\title{
Retinal Rosette
}

National Cancer Institute

\section{Source}

National Cancer Institute. Retinal Rosette. NCI Thesaurus. Code C161565.

Focal to multifocal rosette-like and tubular structures expanding and distorting the inner and outer nuclear layers. (INHAND) 